\title{
Металогический плюрализм и универсальная логика ${ }^{1}$
}

\author{
В. Л. ВАСЮКОВ
}

\begin{abstract}
Conception of logical pluralism claims that there is not one true logic but there are many. Conception of metalogical pluralism is based on the assumption that there is not one correct answer as to whether a given argument is deductively valid, but there are many. Since that leads to the interplay between logics and metalogics the question arises: what is the nature of this interplay? The Universal Logics approach gives us hints at some answers to this question. There are also some semantic keys to the issue under consideration.
\end{abstract}

Ключевые слова: логический плюрализм, логический монизм, логическое следование, общезначимость, комбинации логик, неклассический универсум

\section{1 Введение}

Современная ситуация в логике характеризуется постоянным увеличением числа неклассических логических систем, и процесс этот, судя по всему, необратим в силу своей природы. На протяжении свыше двух тысяч лет исследователи имели дело лишь с логикой Аристотеля и логикой стоиков, и современная классическая логика в значительной степени следует их традиции, отличаясь лишь своим инструментарием. Появление неклассической логики нанесло серьезный удар по логическим исследованиям, заставляя переоценивать и подвергать сомнению многие результаты, считавшиеся доселе незыблемыми. Как следствие, в современной философии логики получила широкое распространение точка зрения о праве на существование не одной, но множества истинных логик. Эта точка зрения получила известность под именем логического плюрализма.

\footnotetext{
${ }^{1}$ Исследование выполнено при финансовой поддержке РГНФ в рамках научно-исследовательского проекта РГНФ («Логический плюрализм и его онтологические и эпистемологические следствия»), проект № 09-03-00545а.
} 
Впрочем, еще Ч. Пирс считал, что существует огромное многообразие логик, которые могут быть определяемы, улучшаемы и используемы для исследования друг друга (или даже самих себя) - все зависит от целей исследователя. В отличие от него для Г. Фреге существовало только одно-единственное исчисление (Begriffsschrift), поскольку всем присуща только лишь одна разновидность человеческого мышления, которую это исчисление отражает. Отсюда и специально разработанный Фреге формальный язык (Formelsprache) - это, по его мнению, не просто какой-то отдельный дополнительный язык, но улучшенная и проясненная версия обычного языка.

В XX веке становление неклассической логики на раннем этапе часто приводило к мирному сосуществованию логического плюрализма и логического монизма в рамках одного и того же философского сообщества. Характерным примером в этом отношении может считаться Львовско-Варшавская логико-философская школа, два выдающихся представителя которой - Станислав Лесьневский и Ян Лукасевич - занимали полярные позиции по вопросу поиска единого основания логики. По мнению Лесьневского, отличие математических систем от произвольных дедуктивных систем вызвано тем, что математические системы не противоречат «логическим интуициям», которые, в свою очередь, не могут произвольно описывать мир. Они в состоянии это делать лишь подчиняясь единой логике - истинной собственной логике мира. Лучше всего, точнее, единственным возможным образом, эту логику можно охарактеризовать как классическую логику - двузначную и экстенсиональную. По этой причине, в частности, Лесьневский не проявлял ни малейшего интереса к многозначным логикам (впрочем и вообще к другим неклассическим логикам).

Что касается Лукасевича, то если в 1936 году он соглашается с Лесьневским, и пишет, что одна и только одна из логических систем реализована в действительном мире, реальна так же как реальна одна и только одна система геометрии, то уже в 1937 году он утверждает нечто противоположеное. Теперь он придерживается той точки зрения, что все логические системы, создаваемые нами, являются при тех допущениях, при которых мы их создаем, необходимо истинными. Можно говорить лишь 
о подтверждении онтологических допущений, скрытых где-то в основании логики, если мы хотим следствия данных допущений проверить как-то на фактах. Наконец, в конце своей жизни в 1952 году он приходит к заключению, что не существует способа распознать, какая из $n$-значных систем логики, $n \geq 2$, истинна. Классическое исчисление высказываний, истинностная матрица которого двузначна, является самой старой и самой простой логической системой и поэтому оно наиболее известно и наиболее широко применяемо. Но для определенных целей, например, в модальной логике, $n$-значная система $(n \geq 2)$ может быть более уместной и применяемой. Чем более применима и богата логическая система, тем более она имеет истинностных значений.

Р. Карнап в своей работе «Логический синтаксис языка» [10] писал, что логика играет огромную роль в философском исследовании, но ничто не заставляет утверждать, что в подобном исследовании мы обязаны иметь дело только с одной логикой. Он формулирует плюралистический «Принцип толерантности», который гласит: наше дело не устанавливать запреты, но получать логические заключения. По его мнению, в логике нет моральных запретов, каждый волен строить и использовать ту логику, которая приносит успех в его исследовании. При этом следует учитывать, что речь, в сущности, идет не о выборе логики, но о выборе соответствующего формального языка.

В наше время, несмотря на то что современная ситуация характеризуется пролиферацией (размножением) неклассических логических систем, что можно было бы считать «эмпирическим» подтверждением правомочности логического плюрализма, дебаты по поводу логического плюрализма не утихают. И главную проблему в связи с этим составляет вопрос о том, являются ли эти логики соперничающими, или же они образуют одно огромное дружное семейство. Такие исследователи, как Грэм Прист, считают, что любая из нестандартных логик (т.е. интуиционистская, многозначная и квантовая, релевантная и паранепротиворечивая, условная и свободная) корректна, их наличие служит нам напоминанием о том, что логика не является множеством принятых истин, но дисциплиной, в которой претендующие на значимость теории соперничают друг с другом. При этом следует различать теоретическую логику, прикладную логику и обы- 
денную логику, ибо области их конкуренции могут не пересекаться.

\section{2 Логический плюрализм и логический монизм}

Какова же природа логического плюрализма с точки зрения современной логики? Карнаповский «принцип толерантности», дающий карт-бланш изобретению и конструированию логических систем, на практике оказался не совсем уместным и приводящим к некоторым недоразумениям. Г. Рестол в [17] показывает, что карнаповская «толерантность», сводящая плюрализм к возможности выбора формальных языков, неверна в том отношении, что в одном и том же языке могут существовать различные отношения логического следования. Поэтому речь должна идти не об отношении между языками, но об отношении между различными видами логического следования.

Последнее весьма существенно, поскольку по мнению Г. Рестола и Дж. Билла [8, p. 475] еще не так давно в логике доминировала точка зрения Фреге-Рассела, согласно которой главным является логическая истина, а логическое следование рассматривалось как вторичное понятие. Может быть, этим и объясняется неудача карнаповского принципа в качестве аргумента в пользу логического плюрализма. Для современной картины логики характерно обратное представление: главное - это логическое следование. Но что тогда представляет собой логическое следование? Что означает, что заключение $A$ следует из посылок $\Sigma$ ? Рестол и Билл считают, что в рамках существующей общепринятой традиции природа логического следования может быть описана следующим образом:

$(\mathrm{V})$ Заключение $A$ следует из посылок $\Sigma$ тогда и только тогда, когда в любом случае, когда каждая посылка из $\Sigma$ истинна, будет истинным $A$. Или равносильно: не бывает так, что каждая посылка из $\Sigma$ истинна, но при этом $A$ не истинно.

Этот принцип позволяет определить общезначимость, например, так, как это делает Р. Джеффри: «Общезначимый вывод есть такой вывод, чье заключение истинно во всех случаях, когда истинны его посылки. Признаком ообщезначимости являет- 
ся отсутствие контрпримеров, случаев, когда все посылки истинны, а заключение ложно» [12, р. 1].

Однако примечательно, что Джеффри заканчивает свое определени замечанием, что трудности с применением этого определения возникают при обсуждении случаев, в нем упомянутых. Вот эти-то трудности следует воспринимать более чем серьезно, ибо они и служат источником логического плюрализма, накладывающего свой отпечаток на всю современную логику, составляя смысл большинства современных исследований.

Подобная специфическая версия логического плюрализма основывается, в сущности, на трех положениях:

- Принцип (V) определяет дотеоретическое (или интуитивное) понятие следования.

- Логика задается уточнением случаев, возникающих в (V). Подобное уточнение может рассматриваться как способ истолкования условий истинности утверждений, выражаемых с помощью рассматриваемого языка.

- Существует по меньшей мере два различных уточнения случаев в (V).

Во втором положении наиболее прозрачное истолкование случаев получается путем простого отождествления их с возможными мирами, столь популярными в современной логике, что приводит к следующему виду определений истинностных значений сложных формул:

$A \wedge B$ истинно в возможном мире $w$ тогда и только тогда, когда $A$ истинно в $w$ и $B$ истинно в $w$.

$A \vee B$ истинно в возможном мире $w$ тогда и только тогда, когда $A$ истинно в $w$ или $B$ истинно в $w$.

$\neg A$ истинно в возможном мире $w$ тогда и только тогда, когда $A$ не истинно в $w$.

$\forall x A(x)$ истинно в возможном мире $w$ тогда и только тогда, когда для каждого объекта $b$ в $w, A(b)$ истинно в возможном мире $w$. 
$\exists x A(x)$ истинно в возможном мире $w$ тогда и только тогда, когда для некоторого объекта $b$ в $w, A(b)$ истинно в возможном мире $w$.

Однако, более стандартным способом в языке классической первопорядковой логики случаи истолковываются в рамках так называемых моделей Тарского. Эти модели представляют собой структуры, включающие в себя следующие конструкции:

1. Непустое множество $D$, называемое областью определения.

2. Функция $I$, интерпретация, удволетворяющая следующим условиям:

(a) $I(E)$ является элементом $D$, если $E$ является именем (в данном языке);

(b) $I(E)$ является множеством упорядоченных $n$-ок $D$-элементов, если $E$ является $n$-местным предикатом.

Использование моделей для определения языка влечет выполнение следующих условий.

- Если $\alpha$ есть приписывание $D$-элементов переменным, то $I_{\alpha}(x)=\alpha(x)$. Если $a$ является именем, то $I_{\alpha}(a)=I(a)$.

- $F t_{1} \cdots t_{n}$ истинно в $M, \alpha$ тогда и только тогда, когда $\left\langle I_{\alpha}\left(t_{1}\right), \ldots I_{\alpha}\left(t_{n}\right)\right\rangle \in I(F)$.

- $A \wedge B$ истинно в $M, \alpha$ тогда и только тогда, когда $A$ истинно в $M, \alpha$ и $B$ истинно в $M, \alpha$.

- $A \vee B$ истинно в $M, \alpha$ тогда и только тогда, когда $A$ истинно в $M, \alpha$ или $B$ истинно в $M, \alpha$.

- $\neg A$ истинно в $M, \alpha$ тогда и только тогда, когда $A$ не истинно в $M, \alpha$.

- $\forall x A$ истинно в $M, \alpha$ тогда и только тогда, когда $A$ истинно в $M, \alpha^{\prime}$ для каждого $x$-варианта $\alpha^{\prime}$.

- $\exists x A$ истинно в $M, \alpha$ тогда и только тогда, когда $A$ истинно в $M, \alpha^{\prime}$ для некоторого $x$-варианта $\alpha^{\prime}$. 
Эти условия дают нам общезначимость аргументов в формальном языке по принципу $(\mathrm{V})$. Аргумент общезначим тогда и только тогда, когда в каждой модели, в которой истинны посылки, будут истинны заключения. Подобное определение можно назвать общезначимостью по Тарскому.

Если же мы будем говорить вместо моделей или миров, например, о ситуациях, то мы получим определение следования и общезначимости в релевантной логике. Если в роли случаев будут выступать вычислительные конструкции (доказательства), то мы получим интуиционистскую логику и т.д. Ясно, что некоторые условия и определения при этом изменяются.

Так, в первом случае условие для отрицания выглядит теперь следующим образом:

- $A$ истинно в $s$ тогда и только тогда, когда $A$ не истинно в $s^{\prime}$, где $s C s^{\prime}$.

Здесь $s C s^{\prime}$ означает, что ситуация $s$ совместима с ситуацией $s^{\prime}$ (т.е. $C$ есть бинарное отношение совместимости). Иными словами, отрицание $A$ истинно в $s$ только когда любая ситуация, в которой $A$ истинно, несовместима с $s$. Принцип $(\mathrm{V})$ в этом случае приобретает ситуационное прочтение:

Вывод $A$ из $\Sigma$ релевантно общезначим, если в любой ситуации, в которой все предпосылки из $\Sigma$ истинны, истинно и $A$.

Для интуиционистской логики существует семантика типа Крипке, в которой истина соотносится с точками (моделирующими вычислительные конструкции), которые частично упорядочены по силе с помощью отношения $\sqsupseteq$. В частности, условия для импликации и отрицания выглядят следующим образом:

- $A \supset B$ истинно в $c$ тогда и только тогда, когда для любой $d \sqsupseteq c$, если $A$ истинно в $d$, то и $B$ также;

- $\neg A$ истинно в $c$ тогда и только тогда, когда $A$ не истинно в $d$ для любой $d \sqsupseteq c$. 
Таким образом конструкция доказывает $A \supset B$ тогда и только тогда, когда будучи скомбинированной с конструкцией для $A$ она дает конструкцию для $B$. Условие для отрицания становится понятным, если учесть, что $\neg A$ определяется как $A \supset \perp$, а для $\perp$ конструкции не существует. Версия принципа $(\mathrm{V})$ для случая конструкций выглядит следующим образом:

Вывод $A$ из $\Sigma$ интуиционистски общезначим, если в любой конструкции, в которой все предпосылки из $\Sigma$ истинны, будет истинно и $A$.

Интуитивно возникающее здесь возражение связано с неопределенностью границ подобных истолкований случаев. Получается, что существует огромное количество отношений следования, порождающих различные логики, и все они несравнимы между собой, в том смысле, что нет никаких объективных критериев, позволяющих судить о превосходстве одного отношения следования над другим. Все они равноправны, следовательно, справедлив принцип «anything goes», т.е. «все дозволено»?

На этот упрек сторонники логического плюрализма обычно отвечают, что задачей их исследований является как раз констатация факта логического плюрализма и прояснение возникающей при подобной констатации картины. Многие из неклассических систем могут быть оспариваемы как по логико-философским, так и техническим причинам, однако это ничего не добавляет к поразительному факту возможности существования множества подобных систем. Собственно говоря, несогласие возникает только тогда, когда уточнены задачи вашей логической системы, установлено, что она должна делать.

Более серьезный аргумент против плюрализма выдвигает Г. Прист в [14, p. 203], рассматривая гипотетический случай двух одинаково хороших определений дедуктивной общезначимости $K_{1}$ и $K_{2}$, когда $\beta$ следует из $\alpha$ в случае $K_{1}$, но не $K_{2}$, и мы знаем, что $\alpha$ истинно. Будет ли $\beta$ истинным, обусловлена ли истинность $\beta$ приводимой информацией? В случе $K_{1}$ это очевидно согласно принципу $(\mathrm{V})$, но вот в случае $K_{2}$ мы сталкиваемся с затруднением. Если считать истинность абсолютным, а не относительным понятием, и учитывать, что общезначимость не означает в общем случае истинность (т.е. в случае $K_{2}$ не 
утверждается, что $\beta$ ложно), то получается, что мы обязаны принять истинность $\beta$, что приводит к тому, что $K_{1}$ информационно предпочтительней $K_{2}$. Отсюда логика, соответствующая $K_{1}$, превосходит логику, соответствующую $K_{2}$.

Дальнейший анализ возникающей в этой связи ситуации приводит С. Рида [16] к заключению, что недостатком подобного плюралистского подхода является принятие классической метатеории, поскольку в этом случае из формализации принципа (V) в виде

$$
\begin{gathered}
\left(\mathrm{V}_{\supset}\right) \Sigma \vdash_{\supset} A \text { тогда и только тогда, когда } \\
(\forall w)((\forall B \in \Sigma) w \vDash B \supset w \vDash A)
\end{gathered}
$$

оказывается, что из $\alpha \vdash_{\supset} \beta$ и истинности $\alpha$ не следует, что $\beta$ истинно. В качестве примера можно взять случай дизъюнктивного силлогизма $(A \vee B, \neg A \vdash B)$, который, как известно (так называемый аргумент Льюиса, см. [15, § 2.6]), приводит к принципу $E x$ Falsum Quodlibet, который не согласуется с (V). Действительно, рассмотрим следующий вывод (см. [9]):

$$
\frac{\frac{\frac{A \wedge \neg A}{A}(\wedge E)}{A \vee B}(\vee I) \quad \frac{A \wedge \neg A}{\neg A}(\wedge E)}{B}(D S)
$$

Получается, что дизъюнктивный силлогизм (с помощью элиминации конъюнкции и введения дизъюнкции) приводит к странному заключению, что все что угодно (в данном случае $B$ ) следует из противоречия (в данном случае $A \wedge \neg A$ ). Это представляется неверным, особенно сторонникам релевантной логики.

Если же классическую метаимпликацию заменить на релевантную, то соответствующая модификация принципа (V)

$$
\begin{aligned}
& \left(\mathrm{V}_{\rightarrow}\right) \Sigma \vdash \rightarrow A \text { тогда и только тогда, когда } \\
& (\forall w)((\forall B \in \Sigma) w \vDash B \rightarrow w \vDash A)
\end{aligned}
$$

гарантирует корректное сохранение истинности и корректный учет общезначимости, включая сюда и классический случай. Отсюда Рид делает вывод, что существует только одна правильная логика и этой логикой может быть только релевантная логика с релевантным метаязыком (т.е. с релевантной металогикой), поскольку именно она является составной частью 
требования сохранения истинности от посылок к заключению: если заключение действительно логически следует из посылок, то эти посылки должны быть релевантны заключению.

\section{3 Проблема неклассических металогик}

Возникающий здесь вопрос связан со следующим обстоятельством. В общем виде формализация принципа (V) выглядит следующим образом:

$$
\begin{aligned}
& \left(\mathrm{V}_{\Rightarrow}\right) \Sigma \vdash_{\Rightarrow} A \text { тогда и только тогда, когда } \\
& (\forall w)((\forall B \in \Sigma) w \vDash B \Rightarrow w \vDash A) \text {. }
\end{aligned}
$$

Рид замечает, что, согласно Рестолу и Биллу, мы получаем различные варианты ' $\vdash$ ' путем варьирования области ' $w$ ' - рассматривая воозможные миры, конструкции, ситуации и т.д. Однако, область ' $w$ ' должна быть универсальной, поэтому различные теории следования скорее можно получить варьированием ' $\Rightarrow$ '. В классической металогике выбор только один - материальная импликация ' $\supset$ '. В релевантной металогике существует две возможности, поскольку релевантная логика четко различает классическую и релевантную импликацию. Собственно говаоря, монизм Рида и основывается на втором выборе - больше, по его мнению, выбирать не из чего.

Однако, должна ли металогика быть обязательно или классической или релевантной? Подобная точка зрения давно уже не является общепринятой. Тот же Г. Прист, рассматривая теорию истины Тарского и его Т-конструкцию, пишет: «... иногда говорят, что теория Тарского должна основываться на классической логике: эта логика требуется для реализации данной конструкции. Подобное утверждение является попросту ложным. Она может основываться на интуиционистской логике, паранепротиворечивой логике, и, фактически, на большинстве логик» $[14$, p. 45].

В этом случае необходимо исследовать проблему неклассических металогик, возникающую в связи с метаязыковой формулировкой $\left(\mathrm{V}_{\Rightarrow}\right)$ логического следования, которую для простоты можно словесно перефразировать следующим образом: из $A$ следует $B$ тогда и только тогда, когда истинность $A$ влечет истинность $B$. Слово «влечет» указывает здесь на мета- 
логическую импликацию, и, следуя Риду, в этом случае можно переформулировать определение следования в виде «из $A$ релевантно следует $B$ тогда и только тогда, когда истинность $A$ релевантно влечет истинность $B »$. В этом случае мы получаем релевантную логику и на метауровне. Но тогда возникает соблазн рассмотрения и статуса формулировок следующего вида: «из $A$ интуиционистски следует $B$ тогда и только тогда, когда истинность $A$ интуиционистски влечет истинность $B », \ll$ « $A$ квантовологически следует $B$ тогда и только тогда, когда из истинности $A$ квантовологически следует истинность $B$ » и т.п.

Действительно, что означает «истинность $A$ интуиционистски влечет истинность $B \gg$ ? Поскольку для интуиционистской логики существует семантика вычислительных конструкций (доказательств), то общезначимость означает здесь истинность во всех метаконструкциях, т.е. наши случаи здесь означают метаконструкции, связанные специфическим отношением порядка. Отсюда принцип (V) (теперь уже метапринцип) здесь привязан к истинности в «интуиционистских» метаконструкциях, специфика которых определяется семантикой интуиционистской металогики.

Точно так же «истинность $A$ квантовологически влечет истинность $B$ » определяется спецификой «квантовых» случаев, которые представляют собой подмножества состояний квантовой системы. Характерным для квантовой логики явлется отсутствие связки импликации, которую, например, в случае системы квантовой логики Р. Гольдблатта [11] заменяет «выводимость», чья интерпретация совпадает с отношением порядка (теоретикомножественным включением) на ортомодулярной решетке подмножеств состояний. В этом случае в принципе $\left(\mathrm{V}_{\Rightarrow}\right)$ метасвязка ' $\Rightarrow$ ' как раз и представляет собой подобную связку, детерминируя истинность соответствующих формул.

Но что интересно: парадоксальным образом монистический аргумент Рида в пользу релевантной логики можно усилить, как раз учитывая возможность «вариьрования» метасвязки ' $\Rightarrow$ '. Если обратиться к семантике релевантной логики $\mathrm{R}$ с тернарным отношением достижимости, то, как известно, мы получаем классическую логику в рамках $\mathrm{R}$, если расширяем систему семантических постулатов за счет постулата $0<a$ [7, с. 396]. Таким 
образом, если рассмотреть множество $K$ ситуаций, обладающих этим дополнительному свойством, то $\left(\mathrm{V}_{\supset}\right)$ получается из $\left(\mathrm{V}_{\rightarrow}\right)$ путем ограничения квантификации по $w$, т.е. дается принципом

$\left(\mathrm{V}_{\supset}\right) \quad \Sigma \vdash_{\supset} A$ тогда и только тогда, когда $(\forall w \in K)((\forall B \in \Sigma) w \vDash B \rightarrow w \vDash A)$.

Более того, если этот же самый постулат добавить к системе постулатов для позитивной релевантной логики (без отрицания), то в качестве результата мы получаем позитивную интуиционистскую логику [7, с.401]. Ограничивая соответствующий квантор до некоторого множества $P$ ситуаций, обладающих требуемым свойством, получаем релевантно-интуиционистскую версию принципа (V) в виде

$$
\begin{array}{cc}
\left(\mathrm{V}_{\supset_{\text {Int }}}\right) \quad \Sigma \vdash_{\supset_{\text {Int }}} A \text { тогда и только тогда, когда } \\
\\
(\forall w \in P)((\forall B \in \Sigma) w \vDash B \rightarrow w \vDash A) .
\end{array}
$$

$\mathrm{C}$ другой стороны, можно получить версию $\left(\mathrm{V}_{\rightarrow}\right)$, основываясь, например, на квантовой логике. Крон, Марич и Вуйосевич [13] показали, что алгебраическая структура релевантных логик (в частности, системы $\mathrm{R}$ ) может быть получена путем введения на ортомодулярной решетке дополнительной операции $\rightarrow$, при этом если $\vdash_{R} A \rightarrow B$, то $v(A) \leq v(B)$, где $v-$ оценка на ортомодулярных решетках, а $\vdash_{R}-$ знак выводимости в системе R. Здесь $A, B$ обязательно должны представлять собой формулы без знака импликации. Однако аксиому дистрибутивности приходится удалить, заменяя ее на $A \wedge(\neg A \vee$ $(A \wedge B)) \rightarrow B$. Отсюда, если ограничить квантификацию по $w$ ситуациями-состояниями, образующими ортомодулярную решетку $O M L$, получаем квантово-релевантную версию принципа $(\mathrm{V})$ :

$$
\begin{array}{ll}
\left(\mathrm{V}_{\rightarrow}^{\rightarrow_{R}}\right) & \Sigma \vdash_{R} A \text { тогда и только тогда, когда } \\
& (\forall w \in O M L)\left((\forall B \in \Sigma) w \vDash B \Rightarrow_{Q} w \vDash A\right) .
\end{array}
$$

На первый взгляд кажется, что $\left(\mathrm{V}_{\supset_{I n t}}\right)$ и $\left(\mathrm{V}_{\rightarrow}^{\rightarrow}\right)$ являются просто следствием нарушения требования универсальности об- 
ласти $w$ (в обоих случаях мы ограничивали область квантификации по $w)$. Однако ничто не заставляет нас, например, в случае $\left(\mathrm{V}_{R}^{\rightarrow}\right)$ вообще отказаться от трактовки $w$ как случаев, рассматривая вместо ситуаций состояния квантовой системы.

Рассмотрим еще один пример получения «смешанного» принципа $(\mathrm{V})$. Для системы бесконечнозначной логики Лукасевича $E_{\aleph_{0}}$ в работе [18] была построена семантика возможных миров с тернарным отношением достижимости. В этой семантике каждой формуле приписывалось значение истинности из обычной матрицы $[0,1]$ для логики Лукасевича и постулаты для отношение следования совпадали с постулатами для релевантной логики $\mathrm{R}$, но возможные миры были линейно упорядочены относительно бинарной упорядоченности $<$, задаваемой с помощью определения $a<b=d_{d e f} R O a b$. Если ограничить возможные миры только теми, в которых рассматриваемые формул принимают значение 0 или 1 , то для такого множества возможных миров $L W E_{\aleph_{0}}$-релевантная версия принципа $(\mathrm{V})$ сводится к следующему принципу:

$$
\begin{array}{ll}
\left(\mathrm{V}_{\rightarrow_{R}}^{\rightarrow_{L}}\right) & \Sigma \vdash_{\rightarrow_{R}} A \text { тогда и только тогда, когда } \\
& (\forall w \in L W)\left((\forall B \in \Sigma) w \vDash B \rightarrow_{L} w \vDash A\right) .
\end{array}
$$

Более того, пополнив список семантических постулатов за счет постулата $0<a$, мы, как и в случае релевантной логики, получаем $E_{\aleph_{0}}$-классическую версия принципа $(\mathrm{V})$ :

$$
\begin{aligned}
\left(\mathrm{V}_{\supset}{ }^{L}\right) & \Sigma \vdash_{\supset} A \text { тогда и только тогда, когда } \\
& (\forall w \in R L W)\left((\forall B \in \Sigma) w \vDash B \rightarrow_{L} w \vDash A\right) .
\end{aligned}
$$

Напрашивающийся здесь вывод сводится к тому, что расширенная «металогическая» версия принципа $(\mathrm{V})$ может сводиться к следующему:

Вывод $A$ из $\Sigma$ общезначим в некоторой логике, если в соответствующей металогике истинность предпосылок из $\Sigma$ влечет истинность заключения $A$.

Вопрос теперь заключается только в том, какова же должна или может быть соответствующая металогика, определяющая 
следование в исходной логике. Собственно говоря, можно рассматривать две металогические версии принципа (V):

Вывод $A$ из $\Sigma$ общезначим в некоторой логике, если в некоторой металогике истинность предпосылок из $\Sigma$ влечет истинность заключения $A$.

Вывод $A$ из $\Sigma$ общезначим в некоторой логике, если во всех металогиках истинность предпосылок из $\Sigma$ влечет истинность заключения $A$.

Вторая версия малореалистична - трудно рассматривать все металогики. Возникает также вопрос, не является ли выбор металогики детерминированным существованием «перевода» из некоторой логики в данную логику, поскольку само существование «смешанных» принципов типа (V) является следствием вмешательства или подмены семантики одной логики на другую семантику, погружения одних семантических концепций в другие, их ограничивающие (что сказывается в ограничении квантификации по случаям). Приходится также задумываться о том, не приводит ли это к ограничению каких-то аргументов в пользу выбора между монистическими (когда логика совпадает с металогикой) и плюралистическими (когда логика и металогика различны концептуально).

И, наконец, внимания требует еще одно обстоятельство, связанное с понятием металогик. Дело в том, что рассмотрение той или иной неклассической метаимпликации способно породить мета-металогическое определение следования. Если отдельно рассмотреть, например, металогическую часть принципа (V), то поскольку в ней речь идет о металогической импликации, то она также требует применения этого принципа. Таким образом, возникает необходимость мета-металогической версии принципа (V) вида

$(\mathrm{V})$ Заключение $A$ следует из посылок $\Sigma$ тогда и только тогда, когда истиннность заключения А следует из истинности посылок $\Sigma$ тогда и только тогда, когда в любом случае, когда истинность каждой посылки из $\Sigma$ истинна, будет истинной истинность заключения $A$. 
C одной стороны, это приводит к дурной бесконечности. $\mathrm{C}$ другой стороны, это напоминает ситуацию в теории истины С. Крипке [6], приводя к следующей формулировке:

$$
\begin{aligned}
& \left(\mathrm{V}_{\overrightarrow{M e t a}}^{\Rightarrow}\right) \quad \Sigma \vdash \Rightarrow A \text { тогда и только тогда, когда } T(B) \vdash \Rightarrow \\
& T(T(A)) \ldots \\
& \quad \text { тогда и только тогда, когда } T(T(B)) \vdash_{\Rightarrow}
\end{aligned}
$$

с соответствующими модификациями $\vdash \Rightarrow$ в случае «смешанного» принципа. В этом случае помимо крипкевского рассмотрения случаев истинности или ложности на соответствующих метауровнях приходится рассматривать еще и истинность на плюралистических вариантах метауровней.

\section{4 Универсальная металогика}

Проблема статуса «смешанных» версий и метаверсий принципа $(\mathrm{V})$ решается очевидным образом в результате конкретного исследования, однако сразу же можно заметить, что в некотором смысле выбор металогики аналогичен выбору неклассического универсума: выбирая ту или иную неклассическую логику в качестве металогики в принципе $\left(\mathrm{V}_{\Rightarrow}^{M e t a}\right)$, мы неявно детерминируем результаты выводимости той или иной формулы метаязыковыми соображениями, не имеющими прямого отношения к данной логической системе. Возможное отношение между металогиками и логиками можно было бы попытаться оценить в перспективе использования понятия глобальных и локальных логик, когда «глобальная логика» означает некоторую металогику, а «локальная логика» означает логическую систему, сформулированную в объектном языке, чьи условия следования однозначно определяются условиями следования в данной металогике, т.е. в глобальной логике. Однако дурная бесконечность в принципе $\left(\mathrm{V}_{\Rightarrow}^{M e t a}\right)$ делает эту перспективу проблематичной, хотя можно принимать некоторые конвенции: в случае, например, «смешанной» версии принципа $\left(\mathrm{V}^{M e t a}\right)$ считать последним некоторый метауровень, после которого ' $\Rightarrow$ ' уже не изменяется.

Если проводить отождествление некоторых формулировок, типа «из $A$ в логике $X$ следует В тогда и только тогда, когда из истинности А в логике $Y_{1}$ следует истинность В» и «из А в 
логике $X$ следует В тогда и только тогда, когда из истинности А в логике $Y_{2}$ следует истинность В», то в этом случае нам потребуется некоторая теория, дающая нам критерии этого отождествления. В этом качестве можно рассматривать универсальную логику (см. [2], [3], [20]), в рамках которой рассматривается взаимная переводимость и комбинируемость всех логических систем. Тогда отождествление или связь рассматриваемых формулировок можно проводить на основании, например, перевода из логики (металогики) $Y_{1}$ в логику (металогику) $Y_{2}$. Результат будет выглядеть, например, следующим образом: «из А в логике $X$ следует В тогда и только тогда, когда из истинности А в логике $Y_{1}$ следует истинность В» справедливо тогда и только тогда, когда «из А в логике $X$ следует В тогда и только тогда, когда из перевода условий истинности А в логике $Y_{2}$ следует перевод условий истинности В». Если подобное отождествление работает для ряда данных переводов, то в этом случае можно говорить о локальном металогическом монизме.

Вместо отождествления можно получать, пользуясь методами универсальной логики, обгединение двух формулировок. В этом случае в объединении $Y_{1} \oplus Y_{2}$ двух логик $Y_{1}$ и $Y_{2}$ объединенное следование $\vdash_{1 \oplus 2}$ определяется с помощью условия:

$$
\begin{gathered}
T_{i}(\Sigma) \vdash_{i} T_{i}(A) \text { влечет } T_{1 \oplus 2}(\Sigma) \vdash_{1 \oplus 2} T_{1 \oplus 2}(A) \text { для всех } \\
T_{i}(\Sigma) \cup\left\{T_{i}(A)\right\} \in Y_{i}(i=1,2),
\end{gathered}
$$

где $T_{i}(A)$ означает истинность $A$ в логике $Y_{i}$. Этот случай можно было бы характеризовать как аддитивныи металогический монизм, если принять во внимание, что мы можем получить единое следование путем объединения всех следований для формулировок приведенного типа, т.е. объединяя все металогики при неизменной логике.

Наряду с этим можно рассматривать и мультипликативный металогический монизм, прибегнув к произведению двух формулировок. В произведении $Y_{1} \otimes Y_{2}$ двух логических систем $\vdash_{1 \otimes 2}$ следование определяется с помощью условия

$$
\begin{gathered}
\left\langle T_{1}\left(\Sigma_{1}\right), T_{1}\left(\Sigma_{2}\right)\right\rangle \vdash_{1 \otimes 2}\left\langle T_{1}\left(A_{1}\right), T_{1}\left(A_{2}\right)\right\rangle \text { влечет } T_{i}\left(\Sigma_{i}\right) \vdash_{i} T_{i}\left(A_{i}\right) \\
\text { для каждого } T_{i}\left(\Sigma_{i}\right) \cup\left\{T_{i}\left(A_{i}\right)\right\} \in Y_{i}(i=1,2) .
\end{gathered}
$$


В этом случае мы получаем произведение всех следований для формулировок рассмотренного типа, т.е. рассматриваем произведение всех металогик при неизменной логике.

И наконец, можно получить коэкспоненииальный металогический монизм, используя коэкспоненциальное логическое следование $\vdash_{1 \Leftarrow 2}$

$$
\begin{gathered}
T_{1}(\Sigma) \vdash_{1 \Leftarrow 2} T_{1}(A) \text { тогда и только тогда, когда } \\
g\left[T_{1}(\Sigma)\right] \vdash_{2} g\left(T_{1}(A)\right) \text { для всех переводов } g: Y_{1} \rightarrow Y_{2},
\end{gathered}
$$

и экспоненииальный металогический монизм, используя экспоненциальное логическое следование $\vdash_{2 \Rightarrow 1}$ :

$$
\begin{gathered}
T_{1}(\Sigma) \vdash_{2 \Rightarrow 1} T_{1}(A) \text { если и только если существуют переводы } \\
h: Y_{2} \rightarrow Y_{1} \text { и } g: Y_{1} \rightarrow Y_{2}, \text { такие, что } \\
\left.h\left(g\left[T_{1}(\Sigma)\right]\right)\right) \vdash_{1} h\left(g\left(T_{1}(A)\right)\right) .
\end{gathered}
$$

Можно попытаться получить и коэкспоненциальный и экспоненциальный монизмы с помощью формулировок «из перевода А в логике $X_{2}$ следует В тогда и только тогда, когда из nеревода условий истинности А в логике $Y_{2}$ следует перевод условий истинности В», используя (ко)экспоненциальное логическое следование и (ко)экспоненциальное металогическое следование одновременно, но на нашем пути встает проблема всеведения увы, мы не в состоянии знать все возможные логики в явном виде, а, следовательно, мы не в силах актуально осуществить все вышеописанные комбинации.

\section{5 Семантические аспекты логического плюрализма}

Стоит заметить, что проблема противостояния логического плюрализма и логического монизма в основном носит характер внутренней дискуссии между логиками по поводу обоснования тех или иных установок по вопросу о логическом следовании, природе логических связок, способах устранении логических парадоксов и т.д. Переход к более широкой перспективе, вызванной рассмотрением чисто онтологических и эпистемологических следствий принимаемых решений, на повестке дня этих дискуссий возникал лишь в контексте философии науки. Однако общее 
рассмотрение подобных аспектов способно привести и к формулировке гибридного «логико-онтологического» плюрализма, вызванного вопросом о природе онтологических допущений формальных языков различных неклассических логик, и к гибридному «логико-эпистемологическому» плюрализму, вызванному необязательностью принятия некоторых подобных допущений, поскольку мы можем их сконструировать из уже имеющихся а это ставит вопрос о их познаваемости.

Что касается конкретных проблем, вызванных металогическим плюрализмом, то следует напомнить, что метаязыковые аспекты первопорядковой классической логики обычно сводятся к интерпретации с помощью моделей (моделей Тарского). Совокупность всех множеств, называемая универсумом множеств, снабжает нас всевозможными разновидностями моделей, требуемых для интерпретации нашей логики. Отсюда, в некотором смысле, первопорядковая логика детерминируется универсумом множеств (моделей).

Меняя определения операций на множествах, интерпретирующих действие логических связок, таким образом, что они будут представлять собой интерпретацию неклассических логических связок, мы получаем возможность интерпретации соответствующей неклассической логики в данном множестве. Возникает ситуация, когда в классическом универсуме (модели классической логики) у нас существует интерпретация неклассической логики. Подобная ситуация типична для семантики неклассической логики. Мы можем освоить в нашем классическом универсуме столько неклассических логик, сколько нам нужно.

Ситуация изменится, если мы возьмем неклассический универсум, то есть модель неклассической логики, а затем введем в нем классические теоретико-множественные операции. В этом случае мы получим интерпретацию классической логики в неклассическом универсуме. Более того, можно продолжить подобное умножение операций путем повторного использования иных неклассических связок, получая новые интерпретации неклассических систем. По сути дела мы сталкиваемся с ситуацией, когда в рамках неклассического универсума существует интерпретация классической логики наряду с другими логическими системами. 
При этом у нас нет никаких аргументов в пользу предпочтения классического или неклассического универсума. Мы можем утверждать самое большее только то, что имеется одна лежащая в основании (глобальная) логика, определяющая и определенная нашим универсумом, в то время как существует множество (локальных) логик, населяющих универсум, не определяемый ими. Глобальность и локальность в подобном контексте являются просто метафорическими маркерами, фиксирующими состояние дел.

Подобными результатами снабжает нас и теория категорий, а именно, теория топосов. Р. Гольдблатт использовал конструкцию топоса функторов из малой категории в категорию множеств $S e t$ для построения категорной семантики интуиционистской логики, в которой алгебра Гейтинга играет роль малой категории. Подобным же образом была использована категория $S e t^{C N}$ функторов из так называемой $C N$-категории (теоретикокатегорный эквивалент алгебры да Косты) в категорию Set. Эта категория также представляет собой топос и полнота паранепротиворечивой системы логики да Косты $C_{1}$ доказывается именно по отношению к подобной разновидности топосов (см. [19]). Аналогичный подход был реализован и для случая релевантной логики R (см. [1]).

Все эти результаты можно оценивать как параллель к первому способу теоретико-множественного построения неклассических универсумов, когда модели неклассической логики строятся «внутри» модели классической логики.

Можно разработать иной теоретико-категорный метод получения неклассического универсума, в котором будут интерпретироваться другие логики (в частности, классическая логика), т.е. построить параллель ко второму способу теоретико-множественного построения неклассических универсумов. С этой целью строятся конкретные категории, подобно топосам передающие структуру определенных неклассических логик, в частности паранепротиворечивой логики да Косты и квантовой логики, так и рассмотрен проект общего метода получения подобных «алгеброзначных» категорий. Если в первом случае речь шла о семантическом погружении неклассических логик в интуиционистский универсум-топос, то во втором случае речь идет о по- 
строении иных неклассических теоретико-категорных универсумов, играющих роль «неклассического» топоса.

В частности, топос да Косты (или потос в терминологии бразильских логиков, выдвинувших подобную идею, но не реализовавших ее) (см. [4]) представляет собой паранепротиворечивый универсум, в котором можно было бы развивать паранепротиворечивую математику, подобно тому, как это делается в топосах для интуиционистской математики. Но если в случае топоса функторов из алгебры да Косты в категорию множеств все случаи паранепротиворечивости возникают лишь как частные конструкции в интуиционистском универсуме, как некоторые локальные артефакты, то в топосе да Косты эта паранепротиворечивость абсолютно органична и более того, она лежит в основе всех конструкций, она глобальна и фундаментальна. Здесь уже классическая математика возникает как артефакт в паранепротиворечивом универсуме, как некоторое локальное отклонение от паранепротиворечивых закономерностей. Так, например, при интерпретации систем $C_{1}-\mathrm{C}_{n}$ приходится использовать неистинностно-функциональную оценку, а истинностнофункциональная оценка характерна только для случая булевых топосов, которые являются теперь всего лишь частным случаем топосов да Косты.

Второй пример - это квантовый топос или квантос [5]. Он представляет собой категорию, которая структурно ориентирована на квантовую логику, но подобна топосу в том отношении, что ее «классификатор подобъектов» имеет структуру ортомодулярной решетки. Каждый квантос содержит в себе булевы топосы и является универсумом для интерпретации квантовой логики.

\section{Литература}

[1] Васюков В.Л. Интерпретация релевантной логики в топосах // Логика и В.Е.К. M., 2004. C. 112-121.

[2] Васюков В.Л. Проблема структуры универсальной логики // Логические исследования. Вып. 13. М.: Наука, 2006. С. 95-114.

[3] Васюков В.Л. Проблема контекста интерпретации в универсальной логике // Логические исследования. Вып. 14. М.: Наука, 2007. С. 105-130.

[4] Васюков В.Л. Потосы для паранепротиворечивой логики // Современная логика: проблемы теории, истории и применения в науке. Материалы Х Общероссийской научн. конференции, СПб. 2008. С. 105-107. 
[5] Васюков В.Л. Онтология квантовой математики // Вестник Российского университета дружбы народов. Серия: Философия. 2009. №3. С. 57-70.

[6] Kрипке C. Очерк теории истины // Сол А. Крипке. Витгенштейн о правилах и индивидуальном языке. М.: Канон +, 2010. С. 206-254.

[7] Роутлей P., Мейер P. Семантика следования // Семантика модальных и интенсиональных логик. М.: Прогресс, 1981. С. 363-423.

[8] Beall J.C. and Restall G. Logical Pluralism // Australasian Journal of Philosophy. 2000. Vol.78. P. 475-493.

[9] Beall J.C. and Restall G. Defending Logical Pluralism // Logical Consequence: Rival Approaches Proceedings of the 1999 Conference of the Society of Exact Philosophy / John Woods and Bryson Brown (editors), Stanmore: Hermes, 2001. P. 1-22.

[10] Carnap R. The Logical Syntax of Language. Little eld, Adams and Co., 1959. translated by Amethe Smeaton.

[11] Goldblatt R. I. Semantic analysis of orthologic // J.Phil.Log. 1974. Vol.3. №1-2. P. 19-35.

[12] Jeffrey R.C. Formal Logic: its scope and its limits. McGraw Hill, Third edition, 1991.

[13] Kron A., Marič Z., Vujosevič S. Entailment and quantum logic // Current Issues in Quantum Logic / Eds. Beltrametti S., Fraassen B. Van. New York, London: Plenum, 1981. P. 193-207.

[14] Priest G. Doubt Truth to be a Liar. Oxford: Clarendon Press, 2008.

[15] Read S. Relevant Logic. Oxford: Blackwell, 1988.

[16] Read S. Monism: the one true logic // A Logical Approach to Philosophy: Essays in Honour of Graham Solomon / ed. D. DeVidi and T. Kenyon, Springer, 2006. P. 193-209.

[17] Restall G. Carnap's Tolerance, Meaning and Logical Pluralism // Journal of Philosophy. 2002. Vol. 99. P. 426-443.

[18] Vasyukov V. L. The Completeness of the Factor Semantics for Łukasiewicz's Infinitevalued Logics // Studia Logica. 1993. Vol. 52. №1. P. 143-167.

[19] Vasyukov V.L. Paraconsistency in Categories // Frontiers of Paraconsistent Logic / D.Batens, C.Mortensen, G.Priest and J.-P. van Bendegem (eds.). Research Studies Press Ltd., Baldock, Hartfordshire, England, 2000. P. 263-278.

[20] Vasyukov V.L. Structuring the Universe of Universal Logic // Logica Universalis. 2007. Vol.1. №2. P. 277-294. 